Imperial/TP/2013/CC/2

\title{
Non-Gaussian signatures of general inflationary trajectories
}

\author{
Jonathan S. Horner \\ Theoretical Physics, Blackett Laboratory, Imperial College, London, SWr 2BZ, UK \\ Carlo R. Contaldi \\ Theoretical Physics, Blackett Laboratory, Imperial College, London, SW7 2BZ, UK and \\ Canadian Institute of Theoretical Physics, 60 St. George Street, Toronto, M5S 3H8, On, Canada \\ (Dated: April 22, 2022)

\begin{abstract}
We carry out a numerical calculation of the bispectrum in generalised trajectories of canonical, single-field inflation. The trajectories are generated in the Hamilton-Jacobi (HJ) formalism based on Hubble Slow Roll (HSR) parameters. The calculation allows generally shape and scale dependent bispectra, or dimensionless $f_{\mathrm{NL}}$, in the out-of-slow-roll regime. The distributions of $f_{\mathrm{NL}}$ for various shapes and HSR proposals are shown as an example of how this procedure can be used within the context of Monte Carlo exploration of inflationary trajectories. We also show how allowing out-of-slow-roll behaviour can lead to a bispectrum that is relatively large for equilateral shapes.
\end{abstract}

\section{INTRODUCTION}

The recent results from Planck satellite have confirmed that the universe is well described by the $\Lambda$ CDM model [1, 2. A cornerstone of this model is the behaviour of the primordial perturbations to the background homogeneous model which seed the formation of structure in the observed universe. The model assumes the perturbations are almost Gaussian and very close to but not exactly independent of scale. The latter statement following from the observational bounds on the scalar-spectral index $n_{s}=0.9603 \pm 0.0073$ [2].

A period of accelerated expansion in the very early universe driven by the potential energy of a slowly evolving scalar field, the inflaton, 3-14] is the most commonly accepted explanation for the near scale invariance of the primordial perturbations on scales larger than the Hubble length. The inflation scenario also explains why the universe is very homogeneous, isotropic and devoid of monopoles. Inflation has been criticised on the grounds of requiring fine tuning 15 18 and alternatives have been proposed (see e.g. 19 23), however none are as simple as the basic inflation scenario involving a single scalar field.

This statement is simultaneously Inflation's greatest strength and weakness since the observational bounds on $n_{s}$ can be satisfied easily by a large selection of potentials defining even the simplest single field model. To pin down the exact model of inflation more precise observations that can constrain higher order statistics of the perturbations will be required. This is particularly important if even more complicated models requiring multiple fields are to be constrained.

A wealth of information could be gained by measuring the non-Gaussianity of the perturbations. If Inflation did occur then the deviations from scale-independence and a pure Gaussian distribution are inherently linked. In the simplest cases both are small and of order the slow-roll parameter $\epsilon$, representing deviations from pure de-Sitter space [24, 25]. Non-Gaussianity is encoded in the bis- pectrum, or 3-point function of the perturbations. The bispectrum has a much richer structure than the power spectrum as it is, in principle, a function of three different scales and therefore contains a lot more information. It may therefore be a very effective tool for breaking the degeneracy of inflationary models. The bispectrum is often parametrised by the dimensionless quantity $f_{\mathrm{NL}}[26$. Most often $f_{\mathrm{NL}}$ is quoted in some limit for the configuration of the mode triangle involved in the 3-point function and in addition it is usually assumed to be very nearly scale invariant. Thus $f_{\mathrm{NL}}$ is usually regarded as a single amplitude for a particular configuration of the 3-point function.

The calculation of $f_{\mathrm{NL}}$ from inflationary models has received a lot of attention in recent years 27. In particular much focus has been placed on models which generate a large value of $f_{\mathrm{NL}}$ yet retain the near scale invariance of the observed power spectrum 28 32. It was hoped that a large $f_{\mathrm{NL}}$ could be observed, potentially confirming any theory matching the amplitude and shape dependence of $f_{\mathrm{NL}}$, or at the very least, ruling out all the models which do not. Unfortunately, this did not happen with the Planck satellite results which showed that $f_{\mathrm{NL}}$ as measured from Cosmic Microwave Background (CMB) anisotropies, is consistent with zero with standard deviation of $\mathcal{O}(10)$ in all "types" of $f_{\mathrm{NL}}[33$. This means the simplest models of inflation are still perfectly consistent with observations.

Despite this, an accurate calculation of $f_{\mathrm{NL}}$ will still be valuable in future as bounds get stronger and stronger. This is particularly important for comparisons with future Large Scale Structure (LSS) surveys that may constrain $f_{\mathrm{NL}} \sim \mathcal{O}(1)$ (see e.g. [34, 35]). Obtaining accurate estimates of the bispectrum and its scale dependence for generic inflationary solutions will be important for these comparisons. This work will require a numerical evaluation of the primordial bispectrum arising from higherorder correlations of the curvature perturbations. The full numerical treatment of the bispectrum has received little attention over the years, most calculations being analytical and relying on various approximations. Most 
numerical work carried out so far has been concerned only with specific potentials with features that are known to result in large non-Gaussianity and still rely on slow-roll approximations to simplify the calculations [36-39].

This paper describes the full numerical calculation of non-Gaussianity for inflationary, single-field trajectories generated in the Hamilton-Jacobi (HJ) formulation [40]. Initial results from this treatment were reported in [41. The numerical treatment allows the calculation of nonGaussianity in cases where the field is not in the slowroll regime, but still in the perturbative regime where the higher-order interaction couplings are still $\ll 1$. It also allows us to calculate the contribution to all possible "shapes" and "types" of non-Gaussianity.

In this framework large ensembles of inflating solutions, or trajectories, can be generated. These are related to a large class of single field potentials and can, in principle, be compared to observations without restrictions on the the model of inflation [42. Here we examine the resulting distribution in various shapes of local type non-Gaussianity and verify the well-known consistency relation for squeezed, single-field inflation [25, 43. We also confirm that the equilateral configuration of the bispectrum follows a similar distribution.

The paper is organised as follows. In Section II we outline the HJ approach and the analytical framework we are using for our computations. In Section III we describe our computational method, recapping the calculation of the power spectrum, followed by the subtleties involved in the calculation of the bispectrum. In Section [V] we outline the main results of the paper and verify them through some simple consistency checks. We discuss our results in Section $\mathrm{V}$.

\section{HAMILTON JACOBI APPROACH TO INFLATIONARY TRAJECTORIES}

We start by briefly reviewing the HJ approach to inflationary trajectories where we consider the Hubble-SlowRoll (HSR) parameters to be the fundamental quantities of interest, as opposed to the frequently used PotentialSlow-Roll (PSR) parameters [0, 44, 46].

If $\phi$ is a monotonic function of time, we can change the independent variable in the Friedmann equations from $t$ to $\phi$ and consider all quantities as functions of $\phi$. The Friedmann equation and the inflaton's equation of motion then take on the following form

$$
\begin{aligned}
\dot{\phi} & =-2 M_{\mathrm{pl}}^{2} H^{\prime}(\phi), \\
{\left[H^{\prime}(\phi)\right]^{2}-\frac{3}{2 M_{\mathrm{pl}}^{2}} H(\phi)^{2} } & =-\frac{1}{2 M_{\mathrm{pl}}^{4}} V(\phi),
\end{aligned}
$$

where overdots and primes denote a derivative with respect to $t$ and $\phi$ respectively, $H$ is the Hubble rate, and $M_{\mathrm{pl}}^{2}=(8 \pi G)^{-1}$. One of the advantages of performing this change of variable is that one can merely pick a function $H(\phi)$ and this will correspond to an exact solution of a corresponding potential $V(\phi)$. It is straightforward to verify that inflation will occur if the following condition holds

$$
\epsilon=2 M_{\mathrm{pl}}^{2}\left[\frac{H^{\prime}(\phi)}{H(\phi)}\right]^{2} \equiv-\frac{\dot{H}}{H^{2}} \equiv \frac{\dot{\phi}^{2}}{2 M_{\mathrm{pl}}^{2} H^{2}}<1 .
$$

This relation is exact, unlike the equivalent expression for the PSR parameter $\epsilon_{V} \propto\left(V^{\prime} / V\right)^{2}<1$ which is only approximate.

We can define an infinite hierarchy of HSR parameters labeled by index $l$

$$
{ }^{l} \lambda=\left(2 M_{\mathrm{pl}}^{2}\right)^{l} \frac{\left(H^{\prime}\right)^{l-1}}{H^{l}} \frac{d^{(l+1)} H}{d \phi^{(l+1)}} .
$$

From these we can define $\eta \equiv{ }^{1} \lambda=-(\ddot{\phi} / H \dot{\phi})$ and $\xi \equiv{ }^{3} \lambda$. The last ingredient required is the number of $e$-foldings $N$ specifying the change in scale factor $a$ during the inflationary phase $\ln (a)=N$. It is useful to relate this to the Hubble rate as

$$
\frac{\mathrm{d} N}{\mathrm{~d} t}=H .
$$

Combining all of these equations produces the following set of differential equations dictating the evolution of the background

$$
\begin{aligned}
& \frac{\mathrm{d} H}{\mathrm{~d} N}=-\epsilon H, \\
& \frac{\mathrm{d} \epsilon}{\mathrm{d} N}=2 \epsilon(\epsilon-\eta), \\
& \frac{\mathrm{d}^{l} \lambda}{\mathrm{d} N}=(l \epsilon-(l-1) \eta)^{l} \lambda-{ }^{l+1} \lambda .
\end{aligned}
$$

This is the most natural set of variables to use when describing a general inflationary trajectory. These equations will be the starting point of our $f_{\mathrm{NL}}$ calculation. The HSR parameters will evolve in time and each particular inflation model with a particular set of initial conditions will correspond to a distinct trajectory in HSRspace. In other words, specifying the HSR parameters at some particular time and solving the system (6) is precisely equivalent to specifying $\phi\left(t_{0}\right), \dot{\phi}\left(t_{0}\right)$, and $V(\phi)$ and solving the Friedmann equations.

The HJ system (6) is an infinite hierarchy of equations that describe all possible background solutions. For the purpose of computing observables the system is usually truncated by fixing ${ }^{l} \lambda=0$ for $l \geq l_{\max }$. The truncated system still describes exact solutions for the background quantities but restricts the space of solutions to a subset of the infinite system.

Relating the HSR picture to a specific model is straightforward for the simplest cases. For example if we set ${ }^{l} \lambda=0$ for all $l>1$ the only remaining non-zero HSR parameters are $\epsilon$ and $\eta$. This implies $H(\phi)=a \phi^{2}+b \phi+c$ is a quadratic function and hence $V(\phi)$ is quartic. If one specifies an initial condition $H_{0}$ this fixes the potential 
$V(\phi)$ up to a constant shift $\phi \rightarrow \phi+C$. This shift will have no impact on observations because the energy scale is specified by $H_{0}$. We can use this symmetry to remove the linear term in $H(\phi)$ and write the potential as

$$
V(\phi)=\frac{\lambda}{4 !} \phi^{4}+\frac{m^{2}}{2} \phi^{2}+\Lambda .
$$

If one specifies $\epsilon_{0}$ and $\eta_{0}$ at the same time as $H_{0}$ this is then equivalent to solving for the model parameters and initial conditions

$$
\begin{aligned}
\phi_{0} & = \pm \frac{\sqrt{2 \epsilon_{0}}}{\eta_{0}} M_{\mathrm{pl}}, \\
\dot{\phi}_{0} & =\mp \sqrt{2 \epsilon_{0}} H_{0} M_{\mathrm{pl}}, \\
\frac{\lambda}{4 !} & =\frac{3 H_{0}^{2} \eta_{0}^{2}}{16 M_{\mathrm{pl}}^{2}} \\
\frac{m^{2}}{2} & =\frac{H_{0}^{2}}{2}\left(3 \eta_{0}-\frac{3}{2} \epsilon_{0}-\eta_{0}^{2}\right), \\
\Lambda & =\frac{2}{27} \lambda M_{\mathrm{pl}}^{4}\left(1+\frac{27}{2} \frac{m^{2}}{\lambda M_{\mathrm{pl}}^{2}}\right)^{2} .
\end{aligned}
$$

Note that although we have three degrees of freedom we cannot specify $\lambda, m^{2}$, and $\Lambda$ independently. This is simply because we have used our freedom in initial condition $\phi_{0}$ to write $H$ as $H(\phi)=a \phi^{2}+c$. This leaves two degrees of freedom to specify $\lambda, m^{2}$ and $\Lambda$. In practice, if one only requires the shape of the potential $V(\phi)$ it is much simpler to solve for $\phi(N), H(N)$, and $\epsilon(N)$ and use the relation

$$
V(\phi)=3 M_{\mathrm{pl}}^{2} H^{2}\left(1-\frac{\epsilon}{3}\right) .
$$

The only remaining information that needs to be specified in the model above is the total number of $e$-foldings $\Delta N$. When integrating the Friedmann equations for a given potential $V(\phi)$ there is no clear way of ensuring inflation ends, or if it provides enough inflation. Inflation ends exactly when $\epsilon=1$. The only constraint on the length of inflation is that it must last at least roughly $60 e$-foldings 22 in order for all scales up to the present Hubble scale to have been inflated to super horizon scales before the deceleration phase of the standard Big Bang picture. Converting this into some length in time necessarily requires some knowledge of $H$ (which may vary significantly over the whole of inflation) so $N$ is clearly the most natural time variable to use. These constraints on inflation are then easy to implement using the HSR parameter system - to ensure inflation ends we choose the initial condition $\epsilon\left(N_{\text {tot }}\right)=1$. To ensure inflation provides enough $e$-foldings we integrate back in time from $N_{\text {tot }} \rightarrow N=0$ where $N_{\text {tot }} \sim 60$. In practice the exact value of $N_{\text {tot }}$ is not known due to uncertainties in the physics of reheating. When generating random trajectories $\Delta N$ can be drawn from a proposal density distribution to account for this uncertainty.
To generate large ensembles of random inflationary trajectories we can then draw the remaining HSR parameters ${ }^{l} \lambda$ at the end of inflation from proposal densities. In the following the proposal densities are uniform over a specified range in each HSR but could also take different forms e.g. normal distribution. The choice of proposal shape and where the boundary conditions are drawn can lead to significant differences in the distributions of the final observable quantities. A number of different choices have been made in the literature [46-49.

It is important to emphasise that the evolution of these trajectories need not have anything to do with inflationary dynamics as $H(\phi)$ can be completely decoupled from the system. One is perfectly able to solve for $\epsilon(N)$, $\eta(N) \ldots$ without mentioning inflation. The key ingredients to connect with inflation are $H(N)$ and $V(\phi)$ or (9), both of which only require an input function $\epsilon(N)$. The HSR parameters themselves, along with their differential equations, only provide an efficient tool for generating valid functions $\epsilon(N)$ which may then be correctly interpreted as inflationary models [49].

\section{A. Monte Carlo generation of HJ trajectories}

The generation of large ensembles of consistent inflationary trajectories in the HJ formalism lends itself to Monte Carlo Markov Chain (MCMC) comparisons of the inflationary model space with observations such as the Planck CMB measurements. The HSR definition is particularly useful since in the slow roll limit the proposal parameters are closely related to the observables such as $n_{s}$, the tensor-to-scalar ratio $r$, running $d n_{s} / d \ln k$ etc. For example, at second order in HSR parameters

$$
\begin{aligned}
n_{s}= & 1-4 \epsilon+2 \eta-2(1+C) \epsilon^{2}- \\
& \frac{1}{2}(3-5 C) \epsilon \eta+\frac{1}{2}(3-C) \xi, \\
r= & 16 \epsilon[1+2 C(\epsilon-\eta)], \\
n_{t}= & -2 \epsilon+(3+C) \epsilon^{2}+(1+C) \epsilon \eta,
\end{aligned}
$$

where $C=4(\ln 2+\gamma)-5$ and $\gamma$ is the Euler-Mascheroni constant [24]. As described below we calculate all observables numerically and use $10-(12)$ for comparison.

Here, we explore the proposal densities for observables resulting from the HJ formalism and including nonGaussianity. The use of the proposal densities for comparison with the data will be explored in [42. As a simple assumption for the proposal densities from which to draw HSR boundary conditions we use uniform distributions in the range

$$
{ }^{l} \lambda=[-1,1] e^{-s l},
$$

for $l>0$ and where $s$ is a suitable suppression factor. Our boundary conditions will be imposed at the end of inflation so $\epsilon=1$ and $N_{\text {tot }}$ is also drawn from a uniform distribution $N_{\text {tot }}=[60,80]$. In our formulation $N$ increases with time so $N \sim 0$ represents the time at which 


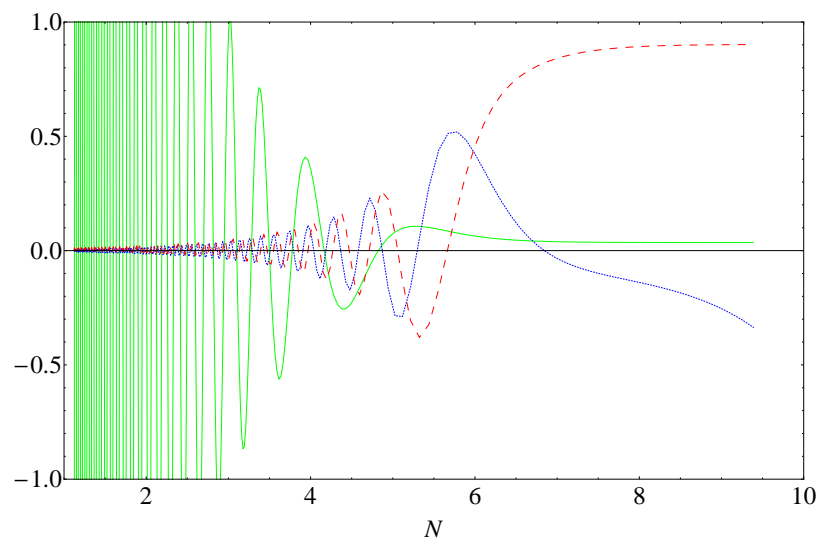

FIG. 1: The evolution of $\zeta$ and $f_{\mathrm{NL}}$ as a function of $e$-fold $N$ for a typical random trajectory. The curves are normalised arbitrarily for the purpose of visualisation. The green (solid) line shows the real part of $\zeta$ for a mode that crosses the horizon at $N \sim 6$. $\zeta$ converges to a constant shortly after horizon crossing as expected. The blue (dotted) and red (dashed) curves show the evolution of the real and imaginary parts of the integral in 47). Only the imaginary part that converges after horizon exit contributes to the value of $f_{\mathrm{NL}}$ whilst the real, diverging component is discarded.

the largest scales observable today were exiting the horizon and $N=N_{\text {tot }}$ is the end of inflation. The observable window spanned by e.g. CMB observations corresponds approximately to the interval $N \sim 0 \rightarrow N \sim 10$. Note that the normalisation of $H$ does not affect the evolution of the parameters so we may specify the initial condition for $H$ at any time in order to correctly normalise the amplitude of perturbations. In practice we have to truncate the HSR series for some finite value of $l=l_{\max }-1$ (so $l_{\max }=3$ implies $\epsilon, \eta, \xi$ are non-zero) ${ }^{1}$.

\section{COMPUTATIONAL METHOD}

\section{A. Computation of the power spectrum}

We introduce a comoving curvature perturbation $\zeta(t, \mathbf{x})$ and work in a gauge where the spatial part of the perturbed metric is given by $g_{i j}=a^{2}(t) e^{2 \zeta(t, \mathbf{x})} \delta_{i j}$ and the inflaton perturbation vanishes everywhere $\delta \phi(t, \mathbf{x})=0$. The primordial power spectrum of the curvature perturbations is related to the variance of the Fourier expanded mode $\zeta_{k}$

$$
\left\langle\zeta_{k_{1}} \zeta_{k_{2}}^{\star}\right\rangle=(2 \pi)^{3} \delta^{(3)}\left(\mathbf{k}_{1}+\mathbf{k}_{2}\right) P_{\zeta}\left(k_{1}\right),
$$

\footnotetext{
1 An alternative "model-independent" method is to parametrise the potential via a Taylor expansion of a certain order as done in [2. The two method are complementary.
}

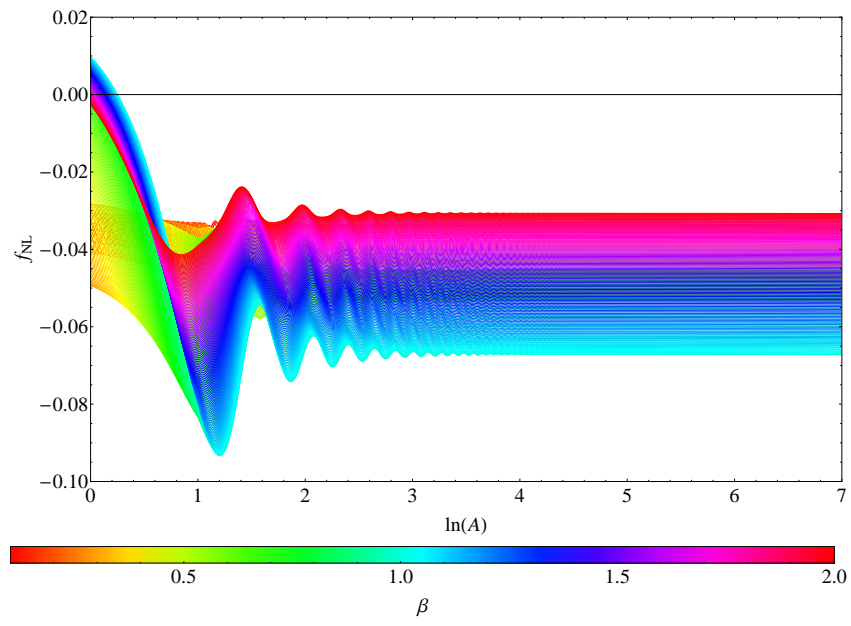

FIG. 2: This figure shows how $f_{\mathrm{NL}}$ for different shape parameter $\beta$ depends on the integration start scale parameter $A$. Each of the curves is generated from the same HSR trajectory for comparison. The parameter $A$ represents how deep inside the horizon the mode smallest $k$ in the triangle was at the start of the integration. $f_{\mathrm{NL}}$ converges for all shapes as $A$ becomes large, signifying earlier start times with respect to horizon exit. Note that, as expected, $f_{\mathrm{NL}}$ peaks at roughly $\beta \sim 1$. Typically when $A \sim 400 f_{\mathrm{NL}}$ has converged with only residual numerical noise at the a level of $\lesssim 1 \%$. The source of the residual noise is the early-time oscillatory integral approximation (see below).

where $\mathbf{k}$ is the Fourier wavevector and $k \equiv|\mathbf{k}|$. The mode $\zeta_{k}(t)$ satisfies the Mukhanov-Sasaki equation [14, 50. Expressed in terms of $N$ instead of $t$ this equation becomes

$$
\frac{\mathrm{d}^{2} \zeta_{k}}{\mathrm{~d} N^{2}}+(3+\epsilon-2 \eta) \frac{\mathrm{d} \zeta_{k}}{\mathrm{~d} N}+\frac{k^{2}}{a^{2} H^{2}} \zeta_{k}=0 .
$$

In this form it is trivial to see that outside the horizon the derivative of $\zeta_{k}$ decays exponentially with respect to $N$ or as $a^{-2}$ so $\zeta_{k}$ quickly goes to a constant. The power spectrum of interest is then related to the freeze-out value of $\zeta_{k}$ on scales $k \ll a H$

$$
P_{\zeta}(k)=\left|\zeta_{k \ll a H}\right|^{2} .
$$

The initial conditions for the solutions to (15) can be set when the mode is much smaller than the horizon $k \gg a H$ and takes on the Bunch-Davies form [51]

$$
\zeta_{k} \rightarrow \frac{1}{M_{\mathrm{pl}}} \frac{e^{-i k \tau}}{2 a \sqrt{k \epsilon}},
$$

where $\tau$ is conformal time defined by $\mathrm{d} N / \mathrm{d} \tau=a H$. From (15) the phase of $\zeta_{k}$ is irrelevant and we only need its rate of change for the initial condition on $\mathrm{d} \zeta_{k} / \mathrm{d} N$ so we never need to explicitly evaluate $\tau$.

For our $f_{\mathrm{NL}}$ calculation we are interested in solving this equation for an observable range of $10^{-5}<k<$ $10^{-1}$ in units of $(\mathrm{Mpc})^{-1}$ for each inflationary trajectory 

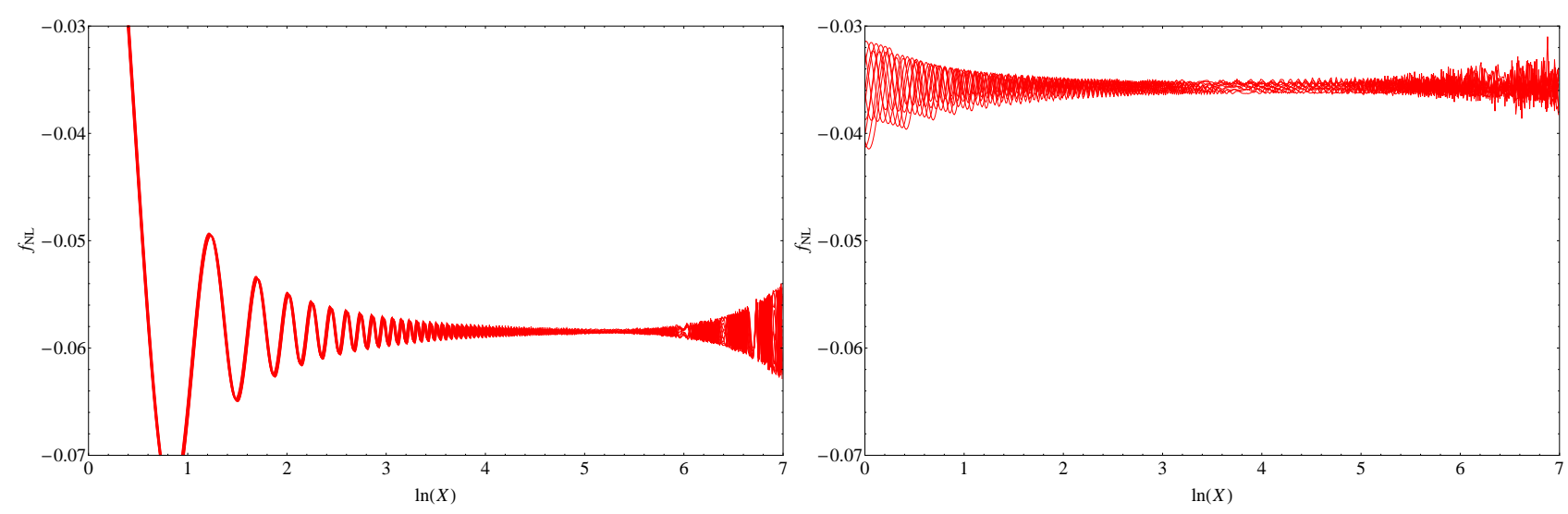

FIG. 3: Left Panel: Dependence of $f_{\mathrm{NL}}$ on the position of the integral split point parameter $X$. The ten lines are for $f_{\mathrm{NL}}$ from 10 "equilateral" shape configurations $(\beta=[0.95,1.05])$ for the same HSR trajectory. If the split point is too late, $X=k / a H \rightarrow 1$ then the WKB approximation used to calculate the early contribution from the diverging, oscillating integrand breaks down. If the split point is too early then inaccuracies in the numerical integration of the oscillatory function start to dominate. The optimal value of the split point is found to be $\ln X=4 \rightarrow 5$ where the total noise is $\ll 1 \%$. Right panel: same but for the ten most "squeezed" triangles (i.e. with $\beta=[0.1-0.2]$ ). The optimal value for $X$ is slightly lower in this case but still small for the choice $\ln X=4 \rightarrow 5$.

obtained via the HJ system. Each background model is completely defined from the solutions of (6) up to an overall normalisation of $H$. To choose this normalisation we need to look at our calculation of $\zeta_{k}$ more closely.

We integrate (15) from a time satisfying $k=A a H$ to $k=B a H$ where $A \gg 1$ and $B \ll 1$ representing sub and super-horizon times respectively. Whatever units we wish to work in, we can fix the normalisation of $a$ so that at $N=0$ the following condition is satisfied

$$
k_{\min }=A a H .
$$

Here $k_{\min }$ represents the smallest $k$ of interest, in practice the mode corresponding to the largest scales observable today. For this particular mode one can then approximate the time of horizon crossing as $N_{c} \approx \ln A$ (this is exact if $H$ is exactly constant and is the only time we use this approximation). The initial condition on $H$ will have a direct effect on the amplitude of the power spectrum. Therefore during the background integration of the flow parameters we fix the initial condition on $H$ to be

$$
H\left(N_{c}\right)=4 \pi \sqrt{2 \pi \epsilon\left(N_{c}\right)} M_{\mathrm{pl}} A_{s},
$$

where $A_{s}$ is the normalisation of the canonical form of the dimensionless primordial curvature perturbation

$$
k^{3} P_{\zeta}(k)=A_{s} k^{n_{s}-1},
$$

and is typically of the order of $10^{-5}$ to reproduce typical density fluctuations amplitudes.

We also need to increase the total number of $e$-folds $N_{\text {tot }} \rightarrow N_{\text {tot }}+\ln A$. If this was ignored, as $A$ increases the mode would start deeper inside the horizon but the initial conditions on the HSR parameters would remain constant. This would effectively change the trajectory so the HSR values at horizon crossing would be different. Shifting the total $e$-folds by $\ln A$ and enforcing $(19)$ ensures that $H$ and the HSR parameters, evaluated at horizon crossing, are independent of $A$ (how deep the modes start inside the horizon). Neglecting these effects would affect the convergence of the power spectrum as $A \rightarrow \infty$.

A simpler way of normalising $H$ would be to specify the initial condition at the end of inflation (with all the other HSR parameters) but that choice is not as physically transparent. In addition, $H$ may vary by orders of magnitudes during the approximately $60 e$-foldings of evolution. This can lead to a large variation in the overall normalisation of the primordial power which can lead to numerical problems if one wishes to use the results as the input to standard boltzmann codes such as CAMB [52].

To be consistent we require 17 to be satisfied for each $k$. Therefore in order for each mode to start "equally deep" inside the horizon we integrate the background forward in time (from $N=0$ ) until $k=A a H$ for every mode of interest. Applying (17) we integrate the background and (15) until each mode crosses the horizon and satisfies $k=B a H$. This ensures the modes have sufficiently converged to their super-horizon values. In practice it was found that, for the calculation of the bispectrum, the solutions converged for $A \approx e^{6}$ and $B \approx 0.1$. Larger values of $A$ significantly added to computational time due to the erratic early time behaviour of $\zeta_{k}$ with no real benefit.

This completely determines the mode evolution and hence their value on super-horizon scales. We can then calculate physical observables such as $n_{s}$ and $r$ from their definitions directly without resorting to any approxima- 

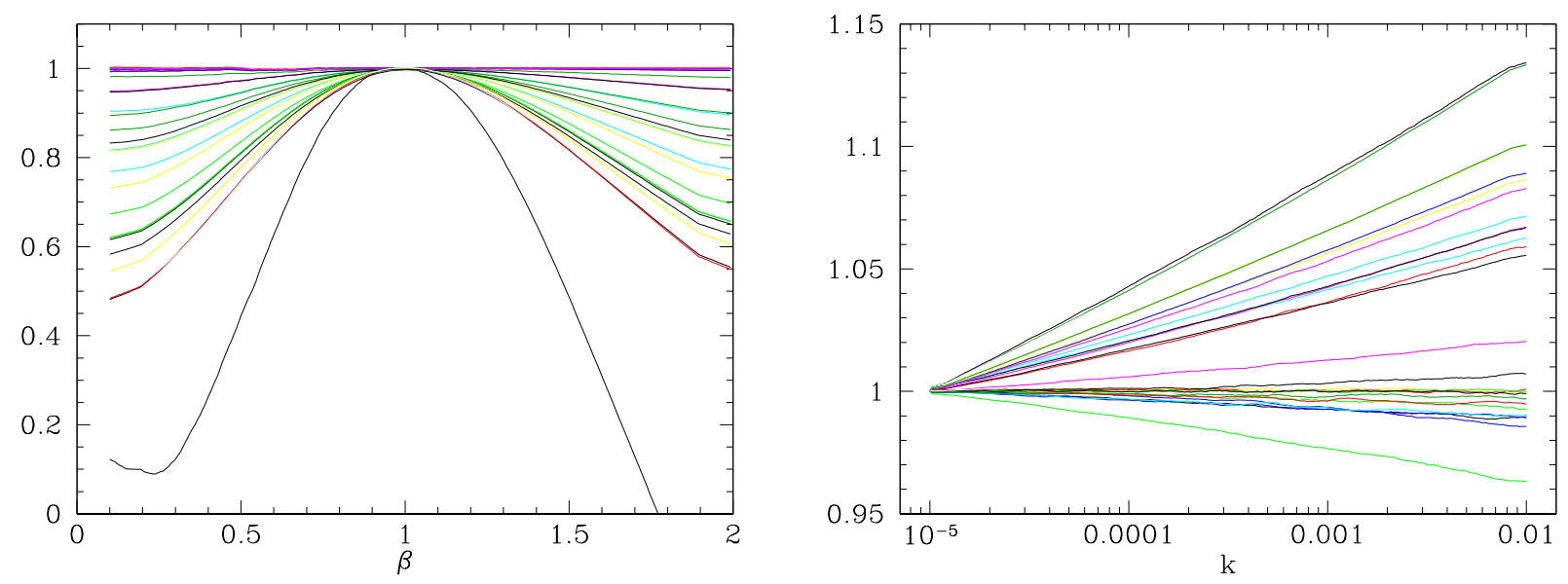

FIG. 4: Shape (left) and scale (right) dependence of $f_{\mathrm{NL}}$ for a selection of trajectories from the "end-of-inflation" boundary condition ensemble. The curves have been normalised with respect to their value at $\beta=1$ and $k_{\star}=10^{-5}(\mathrm{Mpc})^{-1}$ respectively.

tions

$$
\begin{aligned}
n_{s}\left(k_{\star}\right) & =1+\frac{\mathrm{d} \ln \left[k^{3} P_{\zeta}\left(k_{\star}\right)\right]}{\mathrm{d} \ln k} \\
r\left(k_{\star}\right) & =2 \frac{P_{h}\left(k_{\star}\right)}{P_{\zeta}\left(k_{\star}\right)}
\end{aligned}
$$

where we evaluate the quantities at a scale $k_{\star}$ normally chosen to be the largest mode in the system. $P_{h}$ is the power spectrum of either the tensor mode polarisations $h_{+}$and $h_{\times}$. The factor of 2 accounts for the fact that in parity invariant models both polarisations contribute the same exact power. Solutions for both gravitational wave polarisations can be obtained by integrating an equation similar to 15

$$
\frac{\mathrm{d}^{2} h_{k}}{\mathrm{~d} N^{2}}+(3-\epsilon) \frac{\mathrm{d} h_{k}}{\mathrm{~d} N}+\frac{k^{2}}{a^{2} H^{2}} h_{k}=0,
$$

with initial condition

$$
h_{k} \rightarrow \frac{1}{M_{\mathrm{pl}}} \frac{e^{-i k \tau}}{a \sqrt{2 k}}
$$

in the limit where $k \gg a H$.

It is worth noting that choosing $B=1$ (terminating exactly at horizon crossing) produces the the best agreement between equations (21) and (10)-(12) and for very small values of $B$ the results can disagree by $\mathcal{O}(\epsilon)$. This is purely because the slow-roll parameters evolve while the power spectrum remains constant and so the slow-roll formula (which is specified at horizon-crossing) ceases to be valid for sufficiently small $B$. This gives us confidence in our numerical results.

It is important to stress that our choice of priors (in particular our choice of location for the priors) typically generates trajectories where the HSR parameters become small during the time we calculate $P_{k}$. But the method outlined above works for arbitrary values of these parameters. We could specify the initial conditions at the beginning of inflation to begin with, easily breaking slow roll, but we cannot guarantee the trajectory will provide enough inflation.

\section{B. Computation of the bispectrum}

The non-Gaussianity of the primordial curvature perturbations is encoded in the third order moment of $\zeta_{k}$ which, in the isotropic limit, is a function of the wavenumbers of three wavevectors forming closed triangles in momentum space

$$
\left\langle\zeta_{k_{1}} \zeta_{k_{2}} \zeta_{k_{3}}\right\rangle=(2 \pi)^{3} \delta^{(3)}\left(\mathbf{k}_{1}+\mathbf{k}_{2}+\mathbf{k}_{3}\right) B\left(k_{1}, k_{2}, k_{3}\right) .
$$

For convenience the bispectrum $B$ is re-written in a dimensionless form $f_{\mathrm{NL}}\left(k_{1}, k_{2}, k_{3}\right)$ by dividing it by different combinations of the squares of the power spectra of the three modes. $f_{\mathrm{NL}}$ is defined in terms of the bispectrum 26]

$$
\begin{aligned}
f_{\mathrm{NL}}\left(k_{1}, k_{2}, k_{3}\right)= & \frac{5}{6} B\left(k_{1}, k_{2}, k_{3}\right) /\left(\left|\zeta_{k_{1}}\right|^{2}\left|\zeta_{k_{2}}\right|^{2}+\right. \\
& \left.\left|\zeta_{k_{1}}\right|^{2}\left|\zeta_{k_{3}}\right|^{2}+\left|\zeta_{k_{2}}\right|^{2}\left|\zeta_{k_{3}}\right|^{2}\right),
\end{aligned}
$$

and the $5 / 6$ factor has been introduced by convention.

The weighting introduced in (25) is often called the "local" type and others have also been used when motivated by the expected signal-to-noise of different shaped triangles in the observations. In particular [33] analysed the data with respect to two additional weightings - equilateral and orthogonal. The limits reported in [33] are $f_{\mathrm{NL}}^{\text {local }}=2.7 \pm 5.8, f_{\mathrm{NL}}^{\text {equil }}=-42 \pm 75, f_{\mathrm{NL}}^{\text {ortho }}=-25 \pm 39$. 
The $f_{\mathrm{NL}}$ function is normally reduced to a single, scale invariant amplitude for a particular shaped triangle, as above. This motivates the different choice of weightings in analysing observations and reporting results. In our case we will consider the $k_{1}, k_{2}, k_{3}$ dependence of $f_{\mathrm{NL}}$ explicitly and the choice of weighting in relating the bispectrum to the dimensionless $f_{\mathrm{NL}}$ is irrelevant. Throughout this work we use 25] as the definition of $f_{\mathrm{NL}}$ even when we take the limit of different shaped triangles.

In order to calculate $f_{\mathrm{NL}}$ the third order correlator of (24) needs to be calculated at late times in the superhorizon limit. To do this we consider the expansion of the action for $\zeta$ at third order which in terms of the HSR parameters can be written as $25,29,30$.

$$
\begin{aligned}
S_{3}= & M_{\mathrm{pl}}^{2} \int d^{4} x\left[a^{3} \epsilon^{2} \zeta \dot{\zeta}^{2}+a \epsilon^{2} \zeta(\partial \zeta)^{2}\right. \\
& \left.-2 a^{3} \epsilon^{2}\left(1-\frac{\epsilon}{4}\right) \dot{\zeta} \partial_{i} \zeta \partial_{i} \partial^{-2} \dot{\zeta}+\frac{a^{3} \epsilon^{3}}{4} \partial^{2} \zeta \partial_{i} \partial^{-2} \dot{\zeta} \partial_{i} \partial^{-2} \dot{\zeta}+a^{3} \epsilon \frac{\mathrm{d}}{\mathrm{d} t}(\epsilon-\eta) \dot{\zeta} \zeta^{2}+2 f(\zeta) \frac{\delta L}{\delta \zeta}\right],
\end{aligned}
$$

where $\partial_{i} \equiv \partial / \partial x_{i}, \partial^{2}$ and $\partial^{-2}$ are the Laplacian and inverse Laplacian operators respectively, and $\delta L / \delta \zeta$ is the equation of motion 15

$$
\frac{\delta L}{\delta \zeta}=a\left(\frac{\mathrm{d}}{\mathrm{d} t}\left(a^{2} \epsilon \dot{\zeta}\right)+H a^{2} \epsilon \dot{\zeta}-\epsilon \partial^{2} \zeta\right)
$$

The function $f(\zeta)$ is

$$
\begin{aligned}
& f(\zeta)=\frac{\epsilon-\eta}{2} \zeta^{2}+\frac{1}{H} \zeta \dot{\zeta}+ \\
& \frac{1}{4 a^{2} H^{2}}\left(-(\partial \zeta)^{2}-\partial^{-2}\left(\partial_{i} \partial_{j}\left(\partial_{i} \zeta \partial_{j} \zeta\right)\right)\right)+ \\
& \frac{\epsilon}{2 H}\left(\partial \zeta \partial \partial^{2} \dot{\zeta}-\partial^{-2}\left(\partial_{i} \partial_{j}\left(\partial_{i} \zeta \partial_{j} \partial^{-2} \dot{\zeta}\right)\right)\right),
\end{aligned}
$$

which gathers terms proportional to the equation of motion $\delta L / \delta \zeta$ that do not contribute to the third order action.

In analytical estimates of $f_{\mathrm{NL}}$ it is helpful to introduce a number of field redefinitions that simplify the calculations by suppressing the terms proportional to $\delta L / \delta \zeta$ explicitly and isolate the dominant contributions to (26) 25, 30. The redefinitions are not strictly required when calculating the contributions numerically and introduce slow-roll approximations which are against the approach being taken here. The approach described below is equivalent but avoids making some assumptions inherent in the slow-roll limit.

We are interested in calculating the bispectrum using the "in-in" formalism. At tree-level this requires the calculation of $25,30,53$,

$$
\left\langle\zeta^{3}(t)\right\rangle=-i \int_{-\infty}^{t} \mathrm{~d} t^{\prime}\left\langle\left[\zeta^{3}(t), H_{\mathrm{int}}\left(t^{\prime}\right)\right]\right\rangle,
$$

where $H_{\text {int }}$, the interaction Hamiltonian, is essentially $S_{3}$ without the integral over time. Each of the terms in $S_{3}$ contribute separately to the correlation (28) and can be considered individually. We are treating $\zeta$ as a quantised curvature perturbation that is expanded in term of a time dependent amplitude and standard momentum space creation and annihilation operators

$$
\zeta(t, \mathbf{x})=\int \frac{\mathrm{d}^{3} \mathbf{p}}{(2 \pi)^{3}}\left(\zeta_{\mathbf{p}}(t) a_{\mathbf{p}}+\zeta_{-\mathbf{p}}^{*}(t) a_{-\mathbf{p}}^{\dagger}\right) e^{i \mathbf{p} \cdot \mathbf{x}} .
$$

Here $\zeta_{\mathbf{p}}(t)$ is by definition the solution of equation 15$)$ or (26) in Fourier space. Therefore any interaction term proportional to (26) will necessarily vanish and give no contribution because we are expanding in terms of the solutions to that equation.

Since $\zeta$ on super-horizon scales converges at late times we should expect both power spectra and bispectra to converge too. This is not obvious from the form of the action (26) as it requires all terms in $S_{3}$ to converge fast enough at late times. After horizon crossing $\dot{\zeta} \propto a^{-2}$ therefore the $a^{3} \zeta \dot{\zeta}^{2}$ terms in $S_{3}$ decay like $a^{-3}$ and $a^{-1}$ at late times respectively. The same is true for the terms involving $\partial^{-2} \dot{\zeta}$. The $a \zeta(\partial \zeta)^{2} \rightarrow a k^{2} \zeta_{k}^{3}$ term grows like $a$ at late times however. This appears problematic but it will turn out that this divergence gives no contribution to $f_{\mathrm{NL}}$ and will ultimately be discarded.

The final term $\propto a^{3} \dot{\zeta} \zeta^{2}$ is problematic. It grows like $a$ at late times and unlike the $a \zeta(\partial \zeta)^{2}$ term we are not be able to disregard it. One may neglect this term if one assumes certain certain conditions ${ }^{2}$ on $\epsilon-\eta$ but this goes against the spirit of the HSR approach.

The HSR approach also requires a more thorough treatment of boundary terms that have previously been assumed to vanish. Several total derivatives arise from integration by parts during the derivation of the action in the form of (26) and while all the total spatial derivatives can be safely ignored, one total time derivative may give a non-vanishing contribution [54. The contribution, in

\footnotetext{
${ }^{2}$ For example if $\epsilon-\eta$ is sufficiently constant as assumed in analytical approximations or if it decays rapidly enough at late times as done in 36. 37.
} 


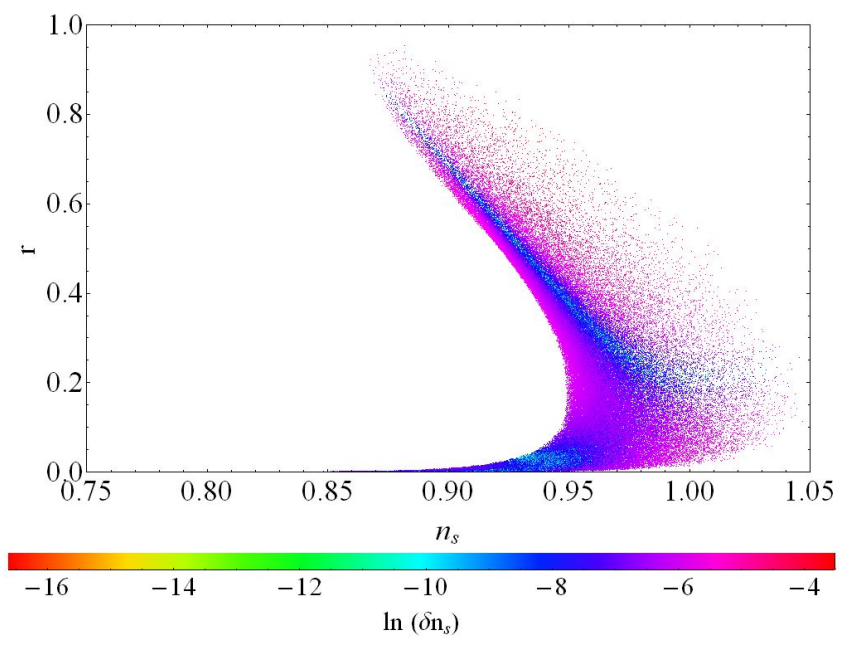

FIG. 5: $r$ vs $n_{s}$ scatter plot for $10^{5}$ trajectories generated as part of the HSR ensemble. Colour represents relative difference from the second order slow-roll formula for $n_{s}$. $k_{*}=10^{-5}(\mathrm{Mpc})^{-1}$. The distribution clearly shows the typical inflationary "attractor" for trajectories with $r>0$.

terms of HSR parameters, is

$$
-\int \mathrm{d}^{4} x \frac{\mathrm{d}}{\mathrm{d} t}\left[(\epsilon-\eta) \epsilon a^{3} \zeta^{2} \dot{\zeta}\right]
$$

Noting the similarity between the boundary term, the apparently divergent $a^{3} \zeta^{2} \dot{\zeta}$ term, and the first term in $f(\zeta)$, we write the final line in 26 as

$$
\begin{array}{r}
\int \mathrm{d}^{4} x\left[a^{3} \epsilon \frac{\mathrm{d} t}{\mathrm{~d} t}(\epsilon-\eta) \zeta^{2} \dot{\zeta}+a(\epsilon-\eta) \frac{\delta L}{\delta \zeta}-\right. \\
\left.\frac{\mathrm{d}}{\mathrm{d} t}\left(a^{3} \epsilon(\epsilon-\eta)\right)+f^{\prime}(\zeta) \frac{\delta L}{\delta \zeta}\right] .
\end{array}
$$

Here the function $f^{\prime}(\zeta)$ contains only derivatives of $\zeta$. It is then straightforward to verify that several cancellations occur in the first three terms resulting in

$$
-2 a^{3} \epsilon(\epsilon-\eta) \zeta \dot{\zeta}^{2}-a \epsilon(\epsilon-\eta) \zeta^{2} \partial^{2} \zeta
$$

The divergent $\zeta^{2} \dot{\zeta}$ disappears in exchange of $\zeta^{2} \partial^{2} \zeta$ which can be dealt with in the same manner as the $\zeta(\partial \zeta)^{2}$ term as described below ${ }^{3}$ We can then finally write the action

\footnotetext{
${ }^{3}$ Note also that the remaining terms proportional to the equation of motion contain only derivatives of $\zeta$ and can be disregarded exactly at the boundary (late times) in the approach taken by 25.
}

as

$$
\begin{aligned}
S_{3}= & \int d^{4} x a^{3} \epsilon\left[(2 \eta-\epsilon) \zeta \dot{\zeta}^{2}+\frac{1}{a^{2}} \epsilon \zeta(\partial \zeta)^{2}\right. \\
& -(\epsilon-\eta) \zeta^{2} \partial^{2} \zeta-2 \epsilon\left(1-\frac{\epsilon}{4}\right) \dot{\zeta} \partial_{i} \zeta \partial_{i} \partial^{-2} \dot{\zeta} \\
& \left.+\frac{\epsilon^{2}}{4} \partial^{2} \zeta \partial_{i} \partial^{-2} \dot{\zeta} \partial_{i} \partial^{-2} \dot{\zeta}\right],
\end{aligned}
$$

where we have dropped terms proportional to the first order equation of motion.

\section{Numerical Calculation of $f_{\mathrm{NL}}$}

Using (33) to define the interaction Hamiltonian one can use equations 28 and 29 to calculate the bispectrum. It can be written in the general form

$$
B\left(k_{1}, k_{2}, k_{3}\right)=\mathcal{I}\left[\zeta_{1}^{*} \zeta_{2}^{*} \zeta_{3}^{*} \int_{N_{0}}^{N_{2}} d N Z(N)\right],
$$

where $\mathcal{I}[z]$ distinguishes the imaginary part of $z, N_{2}$ and $N_{0}$ are defined $e$-folds (times) defined such that all modes are deep inside and far outside the horizon respectively (using the previously defined $A$ and $B$ parameters), $\zeta_{i}=$ $\zeta_{k_{i}}$. There is a contribution to $Z(N)$ for each term in the action. For example, the $\zeta(\partial \zeta)^{2}$ and $\zeta^{2} \partial^{2} \zeta$ terms give the following contribution

$$
\begin{array}{r}
\frac{10}{3 H}\left[a \epsilon^{2}\left(\mathbf{k}_{1} \cdot \mathbf{k}_{2}+\mathbf{k}_{1} \cdot \mathbf{k}_{3}+\mathbf{k}_{2} \cdot \mathbf{k}_{3}\right)+\right. \\
\left.a \epsilon(\eta-\epsilon)\left(k_{1}^{2}+k_{2}^{2}+k_{3}^{2}\right)\right] \zeta_{1} \zeta_{2} \zeta_{3} .
\end{array}
$$

From (33), these are the only terms which do not obviously converge. However, we know at late times $\zeta_{k} \rightarrow$ $A_{k}+\frac{B_{k}}{a^{2}}$ for some $k$-dependant constants. Considering the case $k=k_{1}=k_{2}=k_{3}$ for simplicity

$$
\zeta_{k}^{* 3} \int d N a \zeta_{k}^{3} \approx|A|^{6} \int d N a+\ldots,
$$

where ... denote terms that converge at late times like $a^{-1}$. Only the real part of this expression diverges and we are only interested in the imaginary part for the bispectrum. Therefore these terms cause no issues at late times, unlike the $a^{3} \zeta^{2} \dot{\zeta}$ term.

We now specialise to the case where $k_{1}=k_{2}=k$ and $k_{3}=\beta k$. This allows us to parametrise most shapes of interest via the parameter $\beta$ separately from the overall scale dependence given by wavenumber $k$. Squeezed, equilateral and folded limits correspond to $\beta=0,1$ and 2 respectively. In terms of this classification we can write down our full expression for $f_{\mathrm{NL}}$ as

$$
\begin{aligned}
f_{\mathrm{NL}} & =\frac{1}{|\zeta|^{2}\left(|\zeta|^{2}+2\left|\zeta_{\beta}\right|^{2}\right)} \times \\
& \mathcal{I}\left[\zeta^{* 2} \zeta_{\beta}^{*} \int_{N_{0}}^{N_{2}} d N f_{1} \zeta^{2} \zeta_{\beta}+f_{2} \zeta \zeta^{\prime} \zeta_{\beta}^{\prime}+f_{3} \zeta_{\beta} \zeta^{\prime 2}\right]
\end{aligned}
$$


where $\zeta=\zeta_{k}, \zeta_{\beta}=\zeta_{\beta k}$ and $\zeta^{\prime}=\mathrm{d} \zeta / \mathrm{d} N$. The functions $f_{i}$ are given by

$$
\begin{aligned}
& f_{1}=\frac{5 k^{2} a \epsilon}{3 H}\left(2+\beta^{2}\right)(2 \eta-3 \epsilon), \\
& f_{2}=-\frac{10 H a^{3} \epsilon}{3}\left[4 \eta+\left(1-\beta^{2}\right) \epsilon+\left(\frac{\beta^{2}}{4}-1\right) \epsilon^{2}\right], \\
& f_{3}=-\frac{5 H a^{3} \epsilon}{3}\left[4 \eta+2\left(\beta^{2}-1\right) \epsilon+\left(\frac{\beta^{2}}{4}-1\right) \beta^{2} \epsilon^{2}\right] .
\end{aligned}
$$

The last remaining difficulty lies with the early time behaviour of the integrand. At very early times $\left(N_{0} \rightarrow\right.$ $-\infty, a \rightarrow 0, A \rightarrow \infty) \zeta$ oscillates very rapidly and has a growing amplitude, but the $f_{\mathrm{NL}}$ integral formally converges. At early times the integrand becomes proportional to

$$
\int_{-\infty}^{N} d N f(H, \epsilon, \ldots)\left(\frac{k}{a H}\right)^{n} e^{-i(2+\beta) \frac{k}{a H}}
$$

for some integer $n$. By rotating slightly into the imaginary plane, $(k / a H) \rightarrow(1-i \delta)(k / a H)$ one can obtain a finite answer independent of the cut-off time. Numerically one cannot integrate to infinity and in it's present form the integral does not converge numerically. To resolve this one can add a damping factor to the integrand (similar to the above procedure) however this tends to systematically underestimate the final integrals and the optimum damping factor $\delta$ differs from mode to mode [36, 37.

A better method is to use the early time approximation for $\zeta$ and then integrate by parts. We are interested in calculating an integral of the form

$$
I=\int_{-\infty}^{N} d N f(N) \zeta^{2} \zeta_{\beta}
$$

Using (17) we can write $\zeta^{2} \zeta_{\beta}$ at early times as

$$
\zeta^{2} \zeta_{\beta} \rightarrow \frac{1}{\Gamma} \frac{\mathrm{d}}{\mathrm{d} N}\left(\zeta^{2} \zeta_{\beta}\right)
$$

where

$$
\Gamma=-\left[i(2+\beta) \frac{k}{a H}+3(1+\epsilon-\eta)\right] .
$$

Inserting this into 40 and integrating by parts yields

$$
I \rightarrow\left[\frac{f(N)}{\Gamma} \zeta^{2} \zeta_{\beta}\right]_{-\infty}^{N}-\int_{-\infty}^{N} d N \frac{\mathrm{d}}{\mathrm{d} N}\left(\frac{f(N)}{\Gamma}\right) \zeta^{2} \zeta_{\beta} .
$$

The resulting integral is now more convergent than before as $1 / \Gamma \rightarrow a H / k$. One can repeat the process until the final integrand converges in the limit $a \rightarrow 0$ and all divergences are transferred to the boundary term. These divergences can be removed by using the same contour as before, but now the terms vanish for any finite $\delta$. The boundary term evaluated at $N=-\infty$ can then be safely ignored.
To apply this procedure to the calculation of $f_{\mathrm{NL}}$ we first split the integral into two parts

$$
\int_{N_{0}}^{N_{2}} d N=\int_{N_{0}}^{N_{1}} d N+\int_{N_{1}}^{N_{2}} d N
$$

where $N_{0}$ and $N_{2}$ are times when $k=A a H$ and $k=$ $B a H$ respectively with $A \gg 1$ and $B \ll 1 . N_{1}$ is any time when (17) is a good approximation for both modes. The late time contribution remains unchanged and we perform the "approximate then integrate by parts" procedure to the early time contribution. The early time contribution, $E$, then takes the form

$$
\begin{gathered}
E=\left.\frac{5 H a^{3} \epsilon}{3(2+\beta)^{3}}\left[B_{1} \Gamma+\cdots+\frac{B_{-4}}{\Gamma^{4}}\right] \zeta^{2} \zeta_{\beta}\right|_{N_{1}}- \\
\int_{N_{0}}^{N_{1}} d N \frac{5 H a^{3} \epsilon}{12(2+\beta)^{3}}\left[\frac{A_{-2}}{\Gamma^{2}}+\cdots+\frac{A_{-6}}{\Gamma^{6}}\right] \zeta^{2} \zeta_{\beta},
\end{gathered}
$$

where $A_{i}$ and $B_{i}$ are polynomials of the HSR parameters and $\beta$. For example

$$
\begin{aligned}
B_{1}= & (2+\beta)^{2}[(4+\beta(2 \beta-3)) \epsilon- \\
& \left.2(2+\beta) \eta+\beta\left(1-\frac{\beta^{2}}{4}\right) \epsilon^{2}\right] .
\end{aligned}
$$

We omit the full list of the complicated polynomials for brevity. The second term in 45) gives a completely negligible contribution to the final value of $f_{\mathrm{NL}}$ as it is roughly a factor of $\Gamma^{3}$ smaller and we are in the regime where $\Gamma>>1$. The early time contribution is therefore given completely by the boundary term in 45 .

This method was first used in 37. However the authors choose to focus on particular inflation models such as those with a feature whereas this paper takes a much more general approach. Dealing with the late time divergence from $\zeta^{2} \zeta^{\prime}$ also received little attention. The best explanation on how to deal with this is in 38 , where the authors demonstrate a fortunate cancellation between the troublesome term and the field redefinition.

Here we explicitly keep all terms to all orders in slowroll. Most of the computational effort is spent dealing with the oscillatory nature of $\zeta$ so not much is gained by a slow-roll approximation. This allows a much broader range of models to be analysed which in turn leads to Monte Carlo treatment in the next section. We do drop the early time integration in 45 but this is an approximation relying on the behaviour of $\zeta$ in the limit $k \gg a H$, not an explicit slow-roll approximation. Finally, to our knowledge, this is the first time the third order action has been presented in the form of $(33)$ and used in a calculation. This form provides a much more efficient way to perform the numerical calculation without having to rely on fortuitous cancellations of terms after the integration.

In summary $f_{\mathrm{NL}}$, to a good approximation with respect to the early time oscillatory integral, is given by 
the following expression

$$
\begin{aligned}
f_{\mathrm{NL}} & =\left[\left.|\zeta|^{2}\left(|\zeta|^{2}+2\left|\zeta_{\beta}\right|^{2}\right)\right|_{N_{2}}\right]^{-1} \times \\
& \mathcal{I}\left[\left.\zeta^{* 2} \zeta_{\beta}^{*}\right|_{N_{2}} \int_{N_{1}}^{N_{2}} d N\left(f_{1} \zeta^{2} \zeta_{\beta}+f_{2} \zeta \zeta^{\prime} \zeta_{\beta}^{\prime}+f_{3} \zeta_{\beta} \zeta^{\prime 2}\right)\right. \\
& \left.+\left.\left.\zeta^{* 2} \zeta_{\beta}^{*}\right|_{N_{2}} \frac{5 H a^{3} \epsilon}{3(2+\beta)^{3}}\left[B_{1} \Gamma+\cdots+\frac{B-4}{\Gamma^{4}}\right] \zeta^{2} \zeta_{\beta}\right|_{N_{1}}\right] .
\end{aligned}
$$

\section{RESULTS}

As a check of our method we have verified that our results converge on super-horizon scales and with respect to early-time integration limits. The first condition is illustrated in Figure 1 for a typical random trajectory drawn from the ensemble generated by our method using the end-of-inflation random boundary conditions on the HSRs. A typical trajectory in these ensembles will be deep in the slow-roll regime when modes of interest cross the horizon. The green line is the real part of $\zeta$ while the red and blue lines represent the real and imaginary parts of $f_{\mathrm{NL}}$ as a function of $N$. $f_{\mathrm{NL}}$ oscillates roughly three times quicker than $\zeta$ as it is proportional to $\zeta^{3}$. The real part diverges due to the $k^{2} a \zeta^{3} / H$ term discussed previously however it does not contribute to the amplitude of the correlator in the in-in formalism and can be safely ignored. The imaginary part (the value of interest) converges when the mode exits the horizon. The results shown in figure 1 does not include the contribution of the boundary term in 47) as it contributes only a constant.

The next step is to verify our results do not depend sensitively on the early time cut-off. Figure 2 shows the dependence of $f_{\mathrm{NL}}$ as the integration is started at earlier and earlier times. The color represents the value of $\beta$, our shape parameter for the $\mathbf{k}_{1}+\mathbf{k}_{2}+\mathbf{k}_{3}$ triangle. The value of $f_{\mathrm{NL}}$ converges for all shapes when the parameter $A$, which sets how much smaller than the horizon the mode with the smallest $k$ in the triangle $\mathbf{k}_{1}+\mathbf{k}_{2}+\mathbf{k}_{3}$ has to be at the start of integration, is approximately 400. This is larger than what would be required for an accurate calculation of the corresponding power spectrum statistic due to the diverging oscillatory behaviour of the terms contributing to the $f_{\mathrm{NL}}$ integration.

It is also important to verify convergence with respect to the choice of integration split point $N_{1}$, or cut-off time, introduced in 44. The choice is parametrised by the variable $X$ defined by $X=k / a H$, again this condition is imposed on the smallest $k$ in the triangle $\mathbf{k}_{1}+\mathbf{k}_{2}+\mathbf{k}_{3} . f_{\mathrm{NL}}$ as a function of $X$ is shown in figure 3 . If $X$ is too small, the split point is too close to the time of horizon exit and the early time approximation used in (41) will not be valid. If $X \sim A \rightarrow \infty$, this is equivalent to (37) i.e. doing no regularisation procedure at all. Therefore if $X$ is too large relative to $A$ one would expect the early time contribution to be unable to compensate for the increasingly divergent integral. This is the origin of the noise seen in figure 3. There is an optimal region for the value of $X$ which minimises the combined contribution from both sources of numerical error. From figure 3 it can be seen that $\ln X \approx 4-5$ is a good choice for "folded" shapes $\beta \rightarrow 2$ (left-panel). There optimal position for the split-point is somewhat shape dependent as shown in the right-panel of figure 3 which shows ten "squeezed" cases for the same HSR trajectory but in both cases for $\ln \sim 4$ the inaccuracies are very small $(\ll 1 \%)$. For the following we chose the values $\ln A=6, \ln X=5$, and $B$, the parameter that sets the required size of the largest $k$ in the $\mathbf{k}_{1}+\mathbf{k}_{2}+\mathbf{k}_{3}$ triangle with respect to the horizon at the end of the integration, is set to 0.01 .

We generate ensembles of trajectories for two different HSR boundary conditions. The first is the "end-ofinflation" setup where the HSR are drawn from uniform distributions with a given range at the end of inflation defined by the time when $\epsilon=1$. The second, "early-time" case is one where the HSR, including $\epsilon$ in this case, are drawn from uniform distributions at the time when the largest scale of interest is crossing the horizon. For this case $\epsilon$ is drawn from the range $[0,0.4]$ and the system is evolved back $\ln A=6 e$-folds to the start of the mode integration and then forward for the required number of total $e$-folds to cover horizon exit of all observables scales.

For both cases we used $l_{\max }=4$ and $s=1.5$ as defined in (13) to impose a hierarchical prior. For the "end-ofinflation" ensemble this choice is wide enough to give a proposal distributions in the observables $n_{s}, r$, etc. that are wider than the current, parametric constraints obtained from the recent Planck analysis 2]. For each trajectory the number of $e$-folds was chosen from a uniform distribution in the range be $N_{\text {tot }}=[60,80]+\ln A$. The factor of $\ln A$ is important to maintain convergence in the limit of $A \rightarrow \infty$ as discussed previously. Each ensemble includes some $\mathcal{O}\left(10^{5}\right)$ trajectories.

In figure 4 we show $f_{\mathrm{NL}}$ as a function of shape parameter $\beta$ and overall scale $k$ for a selection 30 trajectories from the "end-of-inflation" ensemble. For this ensemble we expect that at the time when observable quantities are evaluated the HSRs are going to be in the deep slow-roll limit with ${ }^{i} \lambda \ll 1$. This is due to the fact that the system is evolved back from the wide proposal at the end of inflation towards a slow-roll attractor at early times when the observable scales are exiting the horizon. The results for this ensemble should therefore agree with the slowroll approximations and consistency conditions. Figure 4 shows that the scale dependence is very mild and that for trajectories where there is shape dependence $\left|f_{\mathrm{NL}}\right|$ peaks close to the equilateral configuration $\beta=1$. It is also known that $f_{\mathrm{NL}}$ should be near scale-invariant in the slow-roll limit and peak in the equilateral configuration. In addition, $f_{\mathrm{NL}}$ must also satisfy the well known consistency condition in the squeezed limit given by $f_{\mathrm{NL}} \approx(5 / 12)\left(n_{s}-1\right)$ [25, 43].

As a consistency check we also make scatter plots for the ensembles in the $n_{s}$ vs $r$ and $n_{s}$ vs $f_{\mathrm{NL}}$ planes. We do this by plotting the values of $n_{s}, r$, and $f_{\mathrm{NL}}$ from 

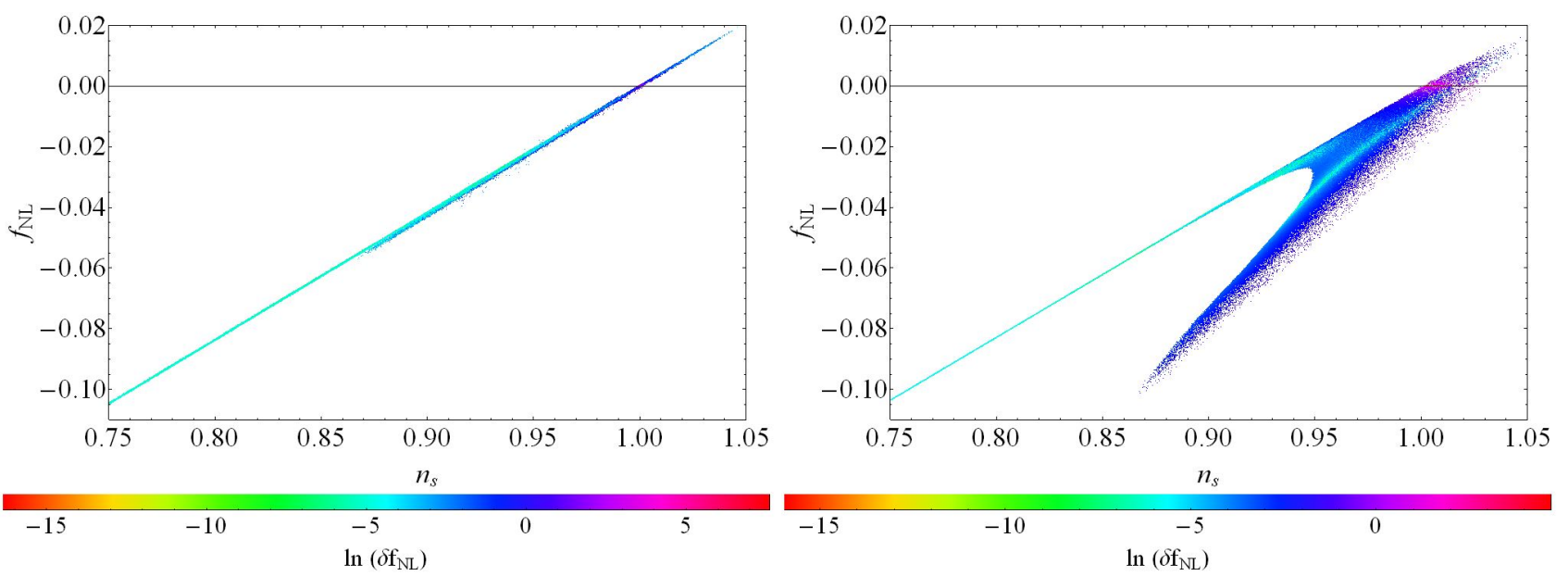

FIG. 6: $n_{s}$ vs $f_{\text {NL }}$ scatter plot for $10^{5}$ trajectories generated with "end-of-inflation" priors. The left panel is for the squeezed limit $\beta=0.1$ and the right panel is for the equilateral case $\beta=1$. The colour scale represents the ln of the relative difference from the slow-roll approximation for $f_{\mathrm{NL}}$. The values of $n_{s}$ and $f_{\mathrm{NL}}$ are sampled for a scale corresponding to $k_{\star}=10^{-5}(\mathrm{Mpc})^{-1}$.

the largest scale for each trajectory in the ensembles. In the slow-roll limit the $n_{s}$ vs $r$ plane should show a clear "inflationary" attractor [40, 45]. The $f_{\mathrm{NL}}$ consistency condition should also appear as a strong attractor in the squeezed $\beta \sim 0$ shape case.

Figure 5 shows the "end-of-inflation" ensemble scatter plot for $n_{s}$ vs $r$. The inflationary attractor is clearly visible. The colour coding in the figure depicts the difference between the numerical $n_{s}$ and second order slow-roll approximation $\bar{n}_{s}$ given by 10 and defined $\delta n_{s}=\left|\left(n_{s}-\bar{n}_{s}\right) / n_{s}\right|$. This shows that the numerical and slow-roll results for $n_{s}$ agree very well when the trajectory lies close to the attractor.

The equivalent of the slow-roll expressions (10)-12 for $f_{\mathrm{NL}}$ is

$$
\bar{f}_{\mathrm{NL}}=\frac{5}{12}\left(\bar{n}_{s}-1+f(\beta) \bar{n}_{t}\right)
$$

where $\bar{n}_{t}$ is the slow-roll approximation for the tensor spectral index and $f(\beta)$ is a function of the shape with $f(\beta) \rightarrow 0$ as $\beta \rightarrow 0$ and $f(\beta)=5 / 6$ when $\beta=1$. Even though this formula was derived only at first order in $\epsilon$, $\eta$ we used the second order formulae for $n_{s}$ and $n_{t}$. Figure 6 shows the trajectories in the $n_{s}$ vs $f_{\mathrm{NL}}$ plane for both the squeezed and equilateral. The $5 / 12 n_{s}$ dependence is clear in both cases but the equilateral case has an additional dependence on $n_{t}$ which dominates when $n_{s} \rightarrow 1$ in analogy with Figure 5 . The figure also shows the difference between the slow-roll approximation for $f_{\mathrm{NL}}$ and the value obtained numerically. The two agree to within a few percent except when $f_{\mathrm{NL}} \ll 10^{-2}$.

Figure 7 shows what happens to the equilateral $f_{\mathrm{NL}}$ distributions in the case where the trajectories are generated using the "early-time" priors on the HSR parameters. In this case, if the proposal ranges for the HSR are wide enough, the largest scales considered will be crossing the horizon when the trajectory is typically still in the out-of-slow-roll regime. At later times the trajectory will typically end up in a slow-roll attractor and the situation will revert to a picture much closer to that seen in figure 6. The squeezed distribution remains unchanged but the equilateral case can have $f_{\mathrm{NL}}$ values much larger than that allowed by the $5 / 12 n_{s}$ scaling. Typically the value of $n_{s}$ for the scale where we are sampling $f_{\mathrm{NL}}$ is also large but we have filtered the trajectories to include only ones where $0.946<n_{s}<0.976$ at the smaller scale $k=10^{-2}(\mathrm{Mpc})^{-1}$ where observational constraints are much tighter. The filter imposes a severe cut on the trajectories with only a fraction $\sim 10^{-3}$ of trajectories satisfying the constraint on $n_{s}$ on smaller scales. For this subset of trajectories the power spectrum, on the largest scales, has a strong scale dependence. This may be preferred by observations of the CMB where there are indications of lower than expected power on the largest scales.

\section{DISCUSSION}

We have outlined a full numerical calculation of the bispectrum of primordial curvature perturbations arising from generalised inflationary trajectories. The bispectrum has been evaluated in terms of a scale dependent $f_{\mathrm{NL}}\left(\mathbf{k}_{1}, \mathbf{k}_{2}, \mathbf{k}_{3}\right)$. The calculation is valid in the out-ofslow-roll regime as long as the weak coupling limit is maintained. This is of interest in models where there is significant evolution of slow-roll parameters during inflation that can lead to observational features in both power spectrum and bispectrum.

We have explored the generation of inflationary ensembles via the HJ formalism using HSR parameters and calculated the distribution of the bispectrum $f_{\mathrm{NL}}$ for various configurations of the $\mathbf{k}_{1}+\mathbf{k}_{2}+\mathbf{k}_{3}$ triangle. In do- 

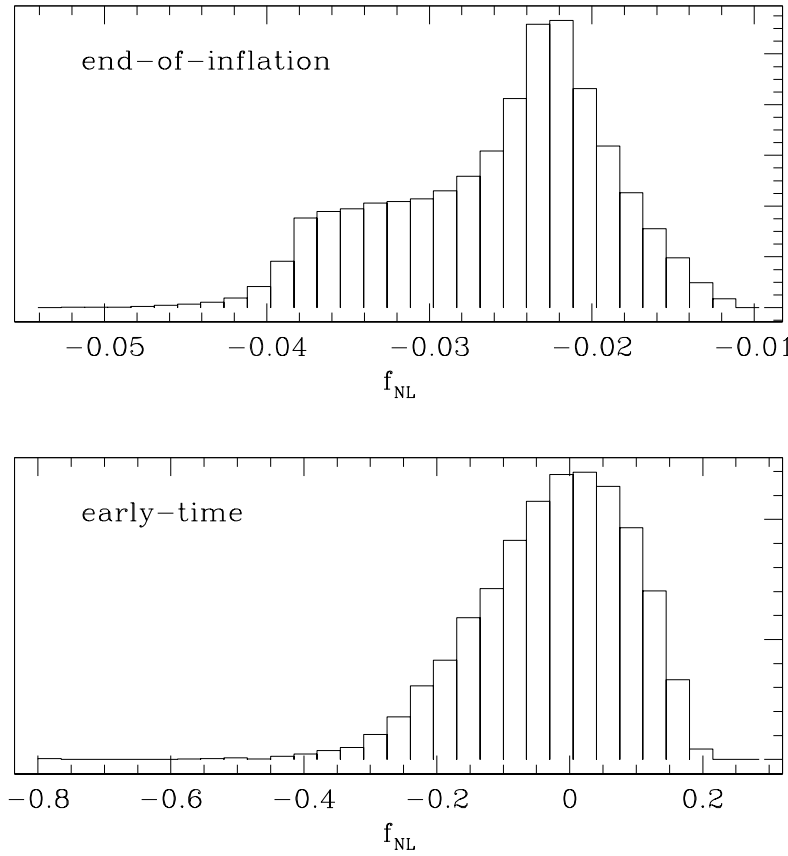

FIG. 7: Histogram of $f_{\mathrm{NL}}$ values equilateral bispectra for the large scale mode $k_{\star}=10^{-5}(\mathrm{Mpc})^{-1}$ in both "end-of-inflation" (top) and "early-time" (bottom) ensembles. Both ensembles have been filtered such that all trajectories have $0.946<n_{s}<$ 0.976 at the smaller scale $k=10^{-2}(\mathrm{Mpc})^{-1}$ in order to agree roughly with observations at the $2 \sigma$ level. The "early-time" proposal of HSR parameters allows for significant variation in the parameters while the largest scales are crossing the horizon leading to $f_{\mathrm{NL}}$ about an order of magnitude larger than in the other case. ing so we have verified the consistency relation for the squeezed limit and the equilateral configurations in the slow-roll regime. We have shown that, in the out-ofslow-roll limit, $f_{\mathrm{NL}}$ equilateral has a much wider distribution due to the scale dependence of the perturbations and has values that are typically an order of magnitude larger than in the slow-roll limit. These types of trajectories can be viable with respect to observations since on smaller scales the perturbations become near scale invariant due to the HSR asymptoting to small values.

The generation of inflationary ensembles including the calculation of the bispectrum will be useful for HSR parameter explorations using future data. $f_{\mathrm{NL}}$ observational constraints are currently far from the regime where they can affect the shape of trajectories and consequently add to our knowledge of the shape of the inflaton potential. However future observations may probe a regime that could constrain any out-of-slow-roll features in the trajectories. This would in turn constrain any significant feature in the single field inflation scenario. Even if features do not exist, probing $f_{\mathrm{NL}}$ to $\mathcal{O}\left(10^{-2}\right)$ by a combination of future LSS observations would be a powerful probe of inflationary physics, particularly in scenarios where no tensor perturbations are detected.

\section{Acknowledgments}

JSH is supported by a STFC studentship. CRC and JSH acknowledge the hospitality of the Perimeter Institute for Theoretical Physics and the Canadian Institute for Theoretical Astrophysics where some of this work was carried out.
[1] P. Ade et al. (Planck Collaboration) (2013), 1303.5062.

[2] P. Ade et al. (Planck Collaboration) (2013), 1303.5082.

[3] A. Starobinsky, Physics Letters B 91, 99 (1980), ISSN 0370-2693, URL http://www.sciencedirect.com/ science/article/pii/037026938090670X

[4] A. H. Guth, Phys. Rev. D 23, 347 (1981), URL http: //link.aps.org/doi/10.1103/PhysRevD.23.347.

[5] A. Albrecht and P. J. Steinhardt, Phys. Rev. Lett. 48, 1220 (1982), URL http://link.aps.org/doi/10.1103/ PhysRevLett.48.1220

[6] A. D. Linde, Physics Letters B 108, 389 (1982).

[7] A. Linde, Physics Letters B 129, 177 (1983), ISSN 03702693, URL http://www.sciencedirect.com/science/ article/pii/0370269383908377

[8] V. F. Mukhanov and G. Chibisov, JETP Letters 33, 532 (1981).

[9] V. Mukhanov and G. Chibisov, Zh. Eksp. Teor. Fiz 83, 487 (1982).

[10] S. W. Hawking, Physics Letters B 115, 295 (1982).

[11] A. H. Guth and S.-Y. Pi, Physical Review Letters 49, 1110 (1982).
[12] A. Starobinsky, Physics Letters B 117, 175 (1982), ISSN 0370-2693, URL http://www.sciencedirect.com/ science/article/pii/037026938290541X

[13] J. M. Bardeen, P. J. Steinhardt, and M. S. Turner, Phys. Rev. D 28, 679 (1983), URL http://link.aps.org/doi/ 10.1103/PhysRevD.28.679.

[14] V. F. Mukhanov, JETP Lett. 41, 493 (1985).

[15] G. Gibbons and N. Turok, Phys.Rev. D77, 063516 (2008), hep-th/0609095.

[16] S. Hollands and R. M. Wald (2002), hep-th/0210001.

[17] L. Kofman, A. D. Linde, and V. F. Mukhanov, JHEP 0210, 057 (2002), hep-th/0206088.

[18] S. Hollands and R. M. Wald, Gen.Rel.Grav. 34, 2043 (2002), gr-qc/0205058.

[19] R. H. Brandenberger, Int.J.Mod.Phys.Conf.Ser. 01, 67 (2011), 0902.4731.

[20] R. H. Brandenberger (2012), 1206.4196.

[21] P. Creminelli, A. Nicolis, and E. Trincherini, JCAP 1011, 021 (2010), 1007.0027.

[22] G. Geshnizjani and N. Ahmadi (2013), 1309.4782.

[23] P. Avelino and R. Ferreira, Phys.Rev. D86, 041501 
(2012), 1205.6676

[24] E. D. Stewart and D. H. Lyth, Phys.Lett. B302, 171 (1993), gr-qc/9302019.

[25] J. M. Maldacena, JHEP 0305, 013 (2003), astro$\mathrm{ph} / 0210603$.

[26] E. Komatsu and D. N. Spergel, Phys.Rev. D63, 063002 (2001), astro-ph/0005036.

[27] N. Bartolo, E. Komatsu, S. Matarrese, and A. Riotto, Phys. Rept. 402, 103 (2004), arXiv:astro-ph/0406398.

[28] K. Tzirakis and W. H. Kinney, JCAP 0901, 028 (2009), 0810.0270

[29] J. Noller and J. Magueijo, Phys.Rev. D83, 103511 (2011), 1102.0275.

[30] D. Seery and J. E. Lidsey, JCAP 0509, 011 (2005), astro$\mathrm{ph} / 0506056$.

[31] E. Silverstein and D. Tong, Phys.Rev. D70, 103505 (2004), hep-th/0310221.

[32] D. Wands, Lect.Notes Phys. 738, 275 (2008), astro$\mathrm{ph} / 0702187$.

[33] P. Ade et al. (Planck Collaboration) (2013), 1303.5084.

[34] N. Dalal, O. Dore, D. Huterer, and A. Shirokov, Phys.Rev. D77, 123514 (2008), 0710.4560.

[35] T. Giannantonio, C. Porciani, J. Carron, A. Amara, and A. Pillepich, Mon.Not.Roy.Astron.Soc. 422, 2854 (2012), 1109.0958.

[36] X. Chen, R. Easther, and E. A. Lim, JCAP 0706, 023 (2007), astro-ph/0611645.

[37] X. Chen, R. Easther, and E. A. Lim, JCAP 0804, 010 (2008), 0801.3295.

[38] D. K. Hazra, L. Sriramkumar, and J. Martin (2012), 1201.0926
[39] H. Funakoshi and S. Renaux-Petel, JCAP 1302, 002 (2013), 1211.3086.

[40] D. S. Salopek and J. R. Bond, Phys. Rev. D 42, 3936 (1990), URL http://link.aps.org/doi/10.1103/ PhysRevD.42.3936

[41] J. S. Horner and C. R. Contaldi (2013), 1303.2119.

[42] C. R. Contaldi and J. S. Horner (in prep.).

[43] P. Creminelli and M. Zaldarriaga, JCAP 0410, 006 (2004), astro-ph/0407059.

[44] P. Adshead and R. Easther, JCAP 0810, 047 (2008), 0802.3898 .

[45] A. R. Liddle, P. Parsons, and J. D. Barrow, Phys.Rev. D50, 7222 (1994), astro-ph/9408015.

[46] W. H. Kinney, Phys.Rev. D56, 2002 (1997), hep$\mathrm{ph} / 9702427$.

[47] R. Easther and W. H. Kinney, Phys.Rev. D67, 043511 (2003), astro-ph/0210345.

[48] S. Chongchitnan and G. Efstathiou, Phys. Rev. D $\mathbf{7 2}$, 083520 (2005), astro-ph/0508355.

[49] A. R. Liddle, Phys.Rev. D68, 103504 (2003), astro$\mathrm{ph} / 0307286$.

[50] M. Sasaki, Prog.Theor.Phys. 76, 1036 (1986).

[51] T. Bunch and P. Davies, Proc.Roy.Soc.Lond. A360, 117 (1978).

[52] A. Lewis and S. Bridle, Phys. Rev. D66, 103511 (2002), astro-ph/0205436.

[53] P. Adshead, R. Easther, and E. A. Lim, Phys.Rev. D80, 083521 (2009), 0904.4207.

[54] F. Arroja and T. Tanaka, JCAP 1105, 005 (2011), 1103.1102 . 